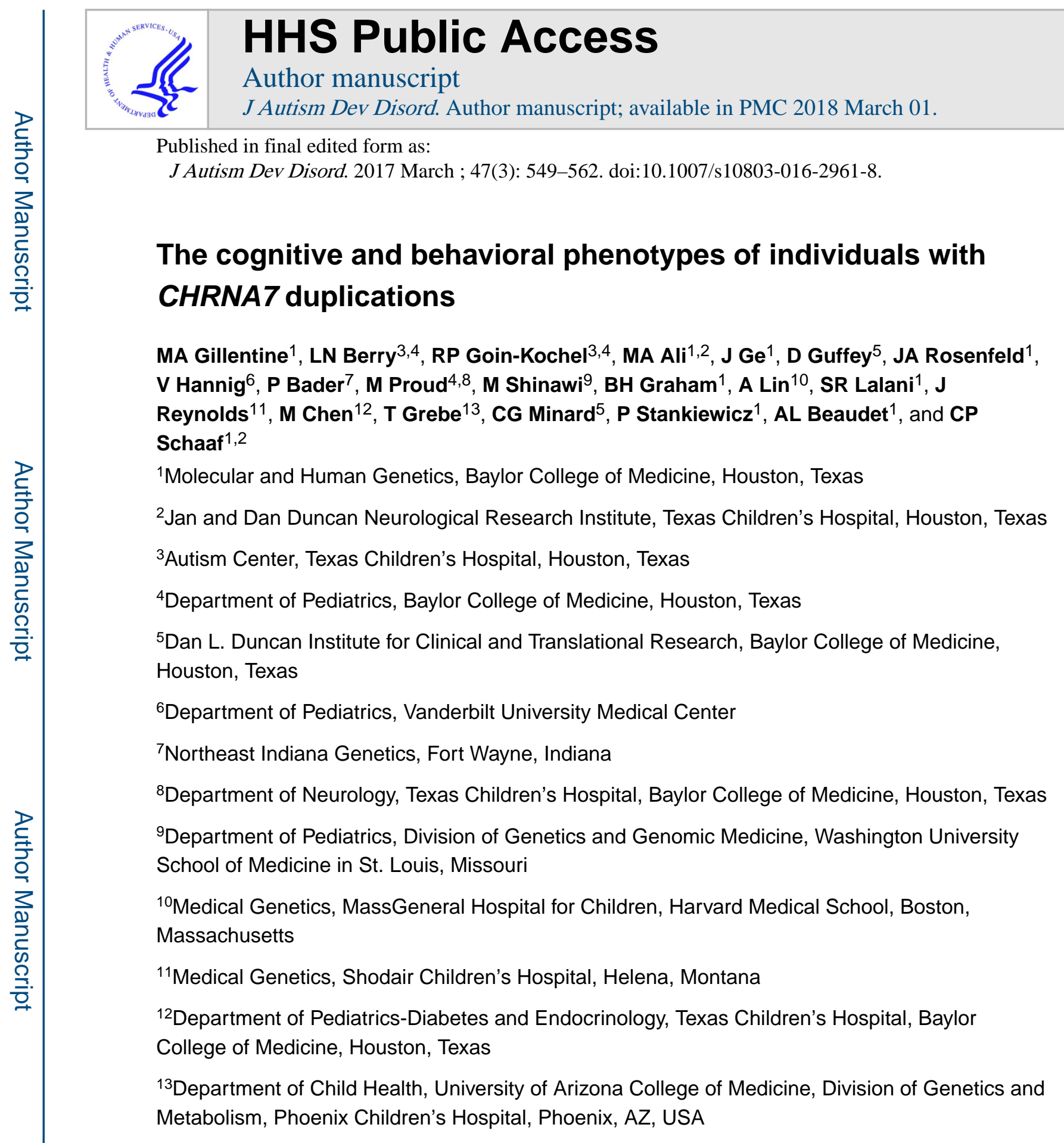

Published in final edited form as:

Autism Dev Disord. 2017 March ; 47(3): 549-562. doi:10.1007/s10803-016-2961-8.

\title{
The cognitive and behavioral phenotypes of individuals with CHRNA7 duplications
}

MA Gillentine ${ }^{1}$, LN Berry ${ }^{3,4}$, RP Goin-Kochel ${ }^{3,4}$, MA Ali $^{1,2}, \mathbf{J ~ G e}^{1}$, D Guffey ${ }^{5}$, JA Rosenfeld ${ }^{1}$, V Hannig ${ }^{6}$, P Bader ${ }^{7}$, M Proud $^{4,8}$, M Shinawi $^{9}$, BH Graham ${ }^{1}$, A Lin ${ }^{10}$, SR Lalani ${ }^{1}, \mathbf{J}$ Reynolds $^{11}$, M Chen ${ }^{12}$, T Grebe ${ }^{13}$, CG Minard ${ }^{5}$, P Stankiewicz ${ }^{1}$, AL Beaudet ${ }^{1}$, and CP Schaaf ${ }^{1,2}$

${ }^{3}$ Autism Center, Texas Children's Hospital, Houston, Texas

${ }^{4}$ Department of Pediatrics, Baylor College of Medicine, Houston, Texas

${ }^{5}$ Dan L. Duncan Institute for Clinical and Translational Research, Baylor College of Medicine,

${ }^{7}$ Northeast Indiana Genetics, Fort Wayne, Indiana

${ }^{8}$ Department of Neurology, Texas Children's Hospital, Baylor College of Medicine, Houston, Texas

${ }^{9}$ Department of Pediatrics, Division of Genetics and Genomic Medicine, Washington University

lof Medicine in St. Louis, Missour

Massachusetts

${ }^{11}$ Medical Genetics, Shodair Children's Hospital, Helena, Montana

${ }^{12}$ Department of Pediatrics-Diabetes and Endocrinology, Texas Children's Hospital, Baylor

Metabolism, Phoenix Children's Hospital, Phoenix, AZ, USA

\section{Abstract}

Corresponding Author Information: Christian P. Schaaf, M.D., Ph.D., Baylor College of Medicine, Department of Molecular and Human Genetics, Jan and Dan Duncan Neurological Research Institute at Texas Children's Hospital, Moursund Street, Ste. 1325, Houston, TX 77030, schaaf@bcm.edu.

Compliance with Ethical Standards:

Ethical approval:

All procedures performed in studies involving human participants were in accordance with the ethical standards of the institutional and/or national research committee and with the 1964 Helsinki declaration and its later amendments or comparable ethical standards. Informed consent: Informed consent was obtained from all individual participants included in the study.

Supplemental Information is available at the Journal of Autism and Developmental Disorders website 
Chromosome 15q11q13 is among the least stable regions in the genome due to its highly complex genomic architecture. Low copy repeat elements at 15q13.3 facilitate recurrent copy number variants (CNVs), with deletions established as pathogenic and CHRNA7 implicated as a candidate gene. However, the pathogenicity of duplications of CHRNA7 is unclear, as they are also found in reportedly healthy parents and unaffected control individuals. We evaluated 18 children with microduplications involving CHRNA7 identified by clinical chromosome microarray analysis (CMA). Comprehensive phenotyping revealed high prevalence of developmental delay/intellectual disability, autism spectrum disorder, and attention deficit/hyperactivity disorder. As CHRNA7 duplications are the most common CNVs identified by clinical CMA, this study provides anticipatory guidance for those involved with care of affected individuals.

\section{Keywords}

15q13.3 microduplication; CHRNA7; neurodevelopment; behavior; autism spectrum disorder

Copy number variation (CNV) at chromosome $15 \mathrm{q} 13$ has been associated with a wide range of clinical neuropsychiatric phenotypes, including intellectual disability (ID), developmental delay (DD), autism spectrum disorder (ASD), epilepsy, and schizophrenia (Gillentine \& Schaaf, 2015). Recurrent CNVs at 15q11q3 result from non-allelic homologous recombination (NAHR) between low copy repeat (LCR) elements that cluster into six breakpoint regions (BP1 to BP6). The largest and least prevalent CNVs are those between BP3 to BP5, spanning over 20 genes (Table 1, Fig. 1) (Gillentine \& Schaaf, 2015). [TABLE 1] Deletions mediated by BP4 and BP5 result in 15q13.3 microdeletion syndrome (OMIM 612001), with the reciprocal duplications being observed at a lower frequency among samples submitted for clinical microarray (CMA) analysis. These BP4-BP5 CNVs contain six genes, FAN1, MTMR10, TRPM1, KLF13, OTUD7A, and CHRNA7, as well as one microRNA: hsa-miR-211. A critical region for these CNVs has been further narrowed by identifying patients with smaller copy number changes between BP4 and BP5 who exhibit similar neurobehavioral features as those caused by the larger CNVs (Shinawi et al., 2009). Smaller 15q13.3 CNVs utilize another LCR element, the distal-CHRNA7-LCR (DCHRNA7-LCR), and BP5 as NAHR substrates. Based on the resolution provided by clinical microarrays, these CNVs appear to encompass the entirety of CHRNA7, and most likely include the first exon of the neighboring gene OTUD7A (Fig. 1) (Shinawi et al., 2009; Szafranski et al., 2010). The clinical variability associated with 15q13.3 CNVs is further complicated both by incomplete penetrance and variable expressivity (Lowther et al., 2015.; Szafranski et al., 2010).

Whereas deletions at 15q13.3 including CHRNA7 have been established as pathogenic, elucidating the pathogenicity of gains involving this gene has proven to be more challenging. It has been previously noted that small gains spanning $C H R N A 7$ with or without the first exon of $O T U D 7 A$ occur in $0.57 \%$ (Gillentine \& Schaaf, 2015) to $0.68 \%$ (Girirajan \& Eichler, 2010; Girirajan et al., 2012) of all samples submitted for clinical CMA, 1.25\% of attention deficit hyperactivity disorder (ADHD) cases (Williams et al., 2012), and 1\% of cases with idiopathic generalized epilepsy (IGE) (Helbig et al., 2009). Of note, the prevalence of 15q13.3 microduplications among control populations has been previously 
reported as 1 in 180 individuals (0.55\%) (Helbig et al., 2009), with some estimates as high as $0.62 \%$ (Girirajan et al., 2012). To date approximately 100 individuals with CHRNA7 gains have been reported, but their clinical and behavioral phenotypes have only been evaluated retrospectively (Gillentine \& Schaaf, 2015). The majority of these reported gains are mediated by the D-CHRNA7-LCR and BP5. Reported phenotypes include ID/DD, ASD, ADHD, and schizophrenia, each in approximately $25 \%$ of affected individuals. While the phenotypes have some overlap with those reported in patients with 15q13.3 deletions, severe cognitive deficits and epilepsy appear to have a lower prevalence among duplication individuals, suggesting that gains of the region may result in a less severe phenotype.

To further delineate the common medical, neuropsychological, and behavioral phenotypes associated with duplications involving the CHRNA7 gene, we enrolled 18 individuals, tested by clinical CMA, to participate in a systematic assessment of their clinical, cognitive, and behavioral phenotypes.

\section{Methods}

This study was approved by the Institutional Review Board at Baylor College of Medicine. Patients were identified retrospectively by review of CMA results performed in the Baylor Miraca Genetics Laboratory (BMGL) or Signature Genomic Laboratory (prior to its closure in 06/2014) and their referring providers were contacted if they had tested positive for 15q13.3 duplications. A total of 18 patients from 17 families (ages 5-14, Fig. 2, Table 2) [TABLE 2] consented to participate in this study and came for a two-day medical and behavioral comprehensive assessment at Texas Children's Hospital. Patients 5 and 17 are brothers, although they were treated independently in statistical analysis. The remaining 16 probands are unrelated.

The clinical indications for CMA in the reported individuals varied (Table 2). Secondary CNVs were detected in five of the 18 study participants (Supplementary Table S1), and reported as findings of uncertain clinical significance on the respective clinical CMA reports for 4 patients. The differing time points of the CMA studies (from 2008 to 2013) performed on each of the participants, and thus differing resolution and interpretation of CNVs, may have affected reporting of secondary CNVs.

Genotypes of all probands were confirmed using genomic DNA isolated from blood samples subjected to multiplex ligation-dependent probe amplification (MLPA) as described previously (Ziats et al., 2016). Parents of each patient who were available and willing to participate were also tested with MLPA to determine CHRNA7 copy number and the inheritance pattern. MLPA reactions were performed according to manufacturer's instructions (MRC Holland). A total of 12 pairs of synthetic probes were designed to detect genes within BP3 to BP5 (Supplementary Table S2).

Probands were evaluated by a team of providers at Texas Children's Hospital. Clinical history and physical exam were obtained by one single provider [C.P.S]. Cognitive and behavioral testing were administered by collaborating psychologists [R.P.K and L.N.B] and included the Differential Ability Scales, Second Edition (DAS-II), the Autism Diagnostic 
Interview-Revised (ADI-R), the Autism Diagnostic Observation Schedule, Second Edition (ADOS-2), the Adaptive Behavior Assessment System, Second Edition (ABAS-II), and the Behavioral Assessment for Children, Second Edition (BASC-2). For some tests, only a subset of probands were tested. This is due to age (Patient 1 for ABAS-II and BASC-2, Patient 6 for subsets of the BASC-2) or due to too low of cognitive functioning (Patient 5 for DAS-II, ADI-R, and ADOS-2). Patient characteristics, IQ measures, and behavioral scales were summarized using frequency with percentage, mean with standard deviation, and median with 25 th and 75 th percentiles. After testing for normality and outliers, a one-sample t-test was used to compare the sample mean to the mean of the normative score as well as to a previously assessed cohort of 15q13.13 deletion probands who were assessed by the same providers at Texas Children's Hospital (Ziats et al., 2016).

\section{Results}

\section{Prevalence of CHRNA7 duplications among CMA samples}

Based on the Baylor Miraca Genetics Laboratories and former Signature Genomics Laboratories, the total number of 15q13.3 microduplications spanning at least D-CHRNA7LCR/BP5 was 567 (of a total of 54407 cases submitted for chromosome microarray analysis, $1.0 \%$ ) at the BMGL (Table 1). At Signature Genomic Laboratory, the total number of 15q13.3 microduplications spanning BP3/BP5 was 3 (of 46 145, 0.007\%), BP4 to BP5 was 35 (of 46 145, 0.76\%), and D-CHRNA7-LCR/BP5 was 213 (of 36 938, 0.58\%). This results in a prevalence of $0.81 \%(1 / 123)$ samples submitted for CMA.

\section{Genetics}

All of the probands in this study had duplications encompassing $C H R N A 7$, ranging from $145 \mathrm{~kb}$ to $3.231 \mathrm{Mb}$ in size (Table 2, Fig 1.). Sixteen patients had approximately $145 \mathrm{~kb}$ duplications mediated by the distal-CHRNA7-LCR (D-CHRNA7-LCR) and BP5. One patient (Patient 16) had a duplication spanning from BP4 to BP5, and one (Patient 4) had a larger gain from BP3 to BP5. All the duplications for which inheritance could be determined $(n=17)$ were inherited (paternal $n=9$, maternal $n=8)$. Over half $(65 \%, n=11)$ of duplications were inherited from parents with self or family reported neuropsychiatric phenotypes, including mood disorders, learning disabilities, ID, ADHD, and schizophrenia. (Figs. 2 and S1, Table 2). Five patients (28\%) had a second CNV outside of the $15 \mathrm{q} 13.3$ region, including four CNVs reported as of uncertain significance and one 22q11.21 deletion (Supplementary Table S1). None of the genes in the CNVs of uncertain significance were present in SFARI Gene or had a neuropsychiatric associated phenotype in the Online Mendelian Inheritance in Man (OMIM).

\section{Clinical Characteristics}

Of the 18 probands, 12 were male and 6 were female (Fig. 3, Table 3). [TABLE 3] The average age was 9.9 years old (SD 3), ranging from 5 to 14 years of age. Only one patient was reported as overweight (weight $>97 \%$ ile), with the rest within normal limits. Three cases each were microcephalic (OFC $<3 \%$ ile) and/or had short stature (height $<3 \%$ ile). Dysmorphic features were present in five patients (28\%), and were more likely to be present 
in probands with additional CNVs, although these were typically mild and not consistent across probands (Figs. 3 and S2).

Developmental delay was common among these probands. On average, patients sat at 7.7 months (range: 5 months to 12 months), crawled at 9.6 months (range: 5 months to 14 months), and walked at 17.2 months (range: 9 months to 36 months) (Supplementary Table S3). Speech delay was typically more severe than motor delay, with probands first word on average at 22.8 months (range: 10 months to 48 months) and two-word sentences at 44.2 months (range: 12 months to 96 months). One patient (Patient 5) was extremely low functioning, resulting in limited cognitive and behavioral evaluation. The most prevalent comorbidity was language or speech delay $(n=11,61 \%)$, with one proband (Patient 5) being nonverbal. Specific speech impairments were not consistent among probands. Sleeping problems were parentally reported, although not formally evaluated, in $28 \%(\mathrm{n}=5)$ of the probands.

\section{Behavioral and Cognitive Phenotype}

Intellectual ability was assessed using the Differential Abilities Scale, Second Edition (DASII) for 17 of the probands (Fig. 4). Figures 4A-C provide histograms of the IQ measure with a curve of the normative scores using a normal distribution with a mean score of 100 and a standard deviation of 15. The average nonverbal ratio IQ (NVRIQ) was 81 (95\% CI: 69.0, 93.2; range 44.2-132) and was lower than the normative average, as were the average verbal ratio IQ (VRIQ) of 79 (95\% CI: 64.1, 94.6; range 24.6-132) and the full scale ratio IQ (FSRIQ) at a mean of 81 (95\% CI: 67.9, 92.5; range 37.7-132). Five (29\%) of the probands who had FSRIQ measurements $(\mathrm{n}=17)$ scored below 70, meeting criteria for ID, while eight (47\%) scored between 70 and 89, meeting criteria for borderline to low average IQ (Table 3, Supplementary Table S5). No significant differences in DAS-II scores were observed between genders (Fig. S3). VRIQ scores differed significantly between nondysmorphic and dysmorphic individuals ( $\mathrm{p}=0.0417$ ), although NVRIQ and FSRIQ scores were not significantly different.

Behavioral phenotypes of 17 probands were assessed using the Adaptive Behavior Assessment System, Second Edition (ABAS-II) and the Behavior Assessment System for Children, Edition 2 (BASC-2). On the ABAS-II, the average General Adaptive Composite (GAC) score was 71.2 (95\% CI: 61.5, 80.9; range: 40-120), considerably lower than the normative average of 100 , with nine individuals scoring extremely low, three below average, four borderline, and one superior (Fig. 5A). The average Conceptual composite score (76.2, 95\% CI: 67.5, 84.9; range: 49-120, 7 extremely low, 6 below average, 2 borderline, 1 average, 1 superior), average Social composite score (79.2, 95\% CI: 70.0, 88.4; range: 54 120, 6 extremely low, 4 below average, 3 borderline, 3 average, 1 superior) and average Practical composite score (72.2, 95\%CI: 61.9, 82.6; range: 40-120, 8 extremely low, 3 below average, 3 borderline, 2 average, 1 superior) were all notably lower than the comparable normative averages (Figs 5B-D). None of the ABAS-II measures were significantly different between genders or by presence of dysmorphic features or additional CNVs (Fig S4). 
On the BASC-2, the average Externalizing T score (50.7; 53.29\%ile; 95\% CI: 45.2, 56.2; range: 37-72) was not outside of the normative range, with only one individual (Patient 13) having a score indicative of clinically significant problems (72) and three patients (Patients 9,11 , and 12 ) having "at risk" scores $(63,65$, and 64 respectively). The average Internalizing T score (50; 47.65\%ile; 95\% CI: 43.1, 56.8; range: 31-82) was also not different from average, with only two individuals (Patients 9 and 13) meeting clinically significant problems with scores of 74 and 82 , respectively (Fig. S4, Supplementary Table S5). However, the average Behavioral Symptoms Index T score of 57.3 (67\% ile; 95\% CI: 50.3, 64.3; range: 42-90) was higher than the normative average, with Patient 9 (score of 84) and Patient 13 (score of 90) meeting clinically significant problems standards and Patients 3 , 11,16 , and 17 within the "at risk" scores between 60 and 69. The average adaptive $\mathrm{T}$ score of 35.9 (14.24\% ile; 95\% CI: 31.3, 40.5; range: 19-47) was significantly lower, with five individuals (Patients 5, 9, 10, 16, and 17) having clinically significantly low scores below 30 (scores of 19, 23, 21, 30, and 30, respectively) and an additional four patients (Patients 8, 11, 13, and 15) with "at risk" scores between 31 and 40 (Fig. 6A-B). CHRNA7 duplication probands had increased average scores on the Hyperactivity (53.76, SD 12.59; 61.24\%ile, SD 31.46; range 31-83), Attention Problems (60.41, SD 10.36; 76.35\%ile, SD 25.46; range: 39-75), Atypicality (58.17, SD 16.25; 67.18\%ile, SD 27.69; range: 41-95), and Withdrawal (60.12, SD 12.61; 73.41\%ile, SD 26.62; range: 41-81) subscales (Figs. 6C, S5, Table S6). Patients had significantly decreased scores on the Leadership (34.69; SD 7.81; 11.69\%ile, SD 10.54; range: 20-46), Activities of Daily Living (34.12, SD 9.02; 11.88\%ile, SD 13.74; range: 16-51), and Functional Communication (35.18, SD 8.09; 13.12\%ile, SD 12.55; range: 18-51) subscales. While the average scores for all of the BASC-2 measures are not of clinical significance (above 70 for clinical measures or below 30 for adaptive measures), they do indicate a trend towards clinical deficits in these categories among CHRNA7 duplication probands.

Patients were assessed for ASD by the Autism Diagnostic Interview-Revised (ADI-R) by the Autism Diagnostic Observation Schedule, Second Edition (ADOS-2), and by clinical impression (Table 3). Of the probands tested for autism spectrum disorder ( $\mathrm{n}=17), 35 \%$ $(n=6)$ had a strict diagnosis of autism spectrum disorder, meeting criteria for both the ADI-R and ADOS-2, all of which also met ASD criteria by clinical impression. Four patients (23.5\%, Patients 8, 9, 11, and 15) and one patient (6\%, Patient 7) met ASD criteria by only the ADI-R or ADOS-2, respectively. One proband (Patient 9) who met ADI-R criteria also met clinical impression criteria for ASD. The patient who met the ADOS- 2 criteria did not met ASD criteria by clinical impression. No significant differences were seen in ASD prevalence by presence of dysmorphic features or by gender (Supplementary Fig. S3). However, the percentage of males (27\%) with ASD was lower than the percentage of females (67\%), however this could be due to the lower number of females in the study. ASD severity and characteristics ranged considerably, as can be seen by the varying ADI-R scores (Fig. 6). A summary of the different ADI-R domains and the ADOS-2 conclusions are in Supplementary Table S5 described both continuously and using the cutoff values.

Additional behavioral phenotypes were reported within this population of patients as diagnosed by primary care providers and reported by parents. ADHD was noted in $44 \%$ $(n=8)$ of probands, consistent with the increased BASC-2 Hyperactivity subscale (Fig. 5G, 
Table 3). Of those with ADHD, two (25\%, Patients 9 and 11) met criteria for autism by the ADI-R and not by the ADOS-2, three (37.5\%, Patients 1,3, and 18) met criteria for both the ADI-R and ADOS-2, and three (37.5\% Patients 4, 12, and 13) did not meet criteria for either test. Other abnormal behaviors, including obsessive-compulsive behaviors and aggression, were parentally reported for $28 \%$ of probands $(n=5)$. Bipolar disorder and/or anxiety were parentally reported in three (17\%) probands, which is consistent with several of the elevated BASC-2 subscale measures.

\section{Comparison to 15q13.3 Deletion Probands}

To further delineate the dosage sensitivity of CHRNA7, we compared our duplication cohort to a recently published 15q13.3 deletion cohort (Ziats et al., 2016), assessed by the same team of investigators. Intellectual disability was present in both cohorts, with duplication probands' DAS-II FSRIQ ( $\mathrm{p}=0.0175)$ and NVRIQ ( $\mathrm{p}=0.0088$ ) measurements being significantly higher than deletion probands' scores (Fig. 8A). All ABAS-II measurements deviated from the average norm in both cohorts. Duplication probands showed a trend of scores deviating less from normal than deletion probands; however this was not statistically significant (Fig. 8B). All BASC-2 scores deviated from the average norm in both groups, although only significantly for a subset of the measures for our cohort of $15 \mathrm{q} 13.3$ duplication individuals. The Externalizing $\mathrm{T}$ score and was significantly lower, and therefore less abnormal, in duplication probands than deletion probands ( $\mathrm{p}=0.0034)$, as were scores for Hyperactivity $(\mathrm{p}=0.0043)$, Aggression $(\mathrm{p}=0.0087)$, and Conduct Problems $(\mathrm{p}=0.0169)$ (Fig. 8C). The Internalizing T score was significantly lower, being closer to the normal population, in duplication probands than deletion probands ( $\mathrm{p}=0.0299)$, with significantly lower scores for Anxiety ( $\mathrm{p}=0.0433)$ and Depression ( $\mathrm{p}=0.0449)$ subscales. The Behavioral Symptoms Index $\mathrm{T}$ score was also significantly lower and deviating less from the normal population in duplication probands ( $\mathrm{p}=0.0311$ ), although none of the sub-scales were substantially different between the two groups. There were no significant differences among BASC-2 adaptive skills.

While duplication probands have higher prevalence of ASD ( $n=6,35 \%)$ and ADHD $(n=8$, $44 \%$ ) relative to the general population, neither was significantly higher than deletion probands ( $\mathrm{n}=5 / 16$ for both ASD and ADHD, 31\%). ADI-R scores also did not differ significantly between $C H R N A 7$ deletion and duplication probands (data not shown).

\section{Discussion}

Chromosomal microduplications of the CHRNA7 gene represent the most common copy number variants identified by clinical CMA, based on the BMGL and formerly Signature Genomics databases. We set out to study the clinical and behavioral phenotypes of 18 children identified to carry duplications involving the CHRNA7 gene.

Common to other copy number variants that predispose to neuropsychiatric disease, $15 \mathrm{q} 13.3$ microduplications exhibit incomplete penetrance and variable expressivity. Incomplete penetrance was observed in this cohort, with $35 \%$ ( $\mathrm{n}=6$ of 17) of $C H R N A 7$ duplications being inherited from a reportedly unaffected parent and Patient 7 having an unaffected sibling carrying a $C H R N A 7$ duplication. Given the variable expressivity, it is important to 
recognize the most prevalent clinical and behavioral features of individuals with CHRNA7 gains. In our group of patients, the variable expressivity is notable in respect to several phenotypes, including IQ measurements. A majority of our cohort have very low to below average IQ (29\% IQ<70, 23.5\% IQ 70-79, 23.5\% IQ 80-89), with FSRIQ scores ranging from 38 (Patient 10) to 132 (Patient 3 ) and the average FSRIQ being 80, suggesting a common feature of ID to borderline ID (Fig. 4). The additional reported behavioral diagnoses and variable ADI-R scores among probands are also reflective of the variable expressivity manifested by these CNVs (Table 3, Fig 6). As has been done with ADHD probands (Williams et al., 2010), choosing subsets of patients with specific phenotypes and determining if $C H R N A 7$ gains are enriched compared to controls may provide further insight into the pathogenicity of these CNVs.

Due to the repetitive elements in this region of the genome and the varying size of CNVs resulting from it, it is logical that the variable expressivity of the phenotypes in patients with $15 q 13.3$ duplications could be due to the differing sizes of the duplications. However, this appears to not be the case. In our small cohort, 16 probands had small CHRNA7-LCR/BP5 duplications, one had a BP4/BP5 duplication (Patient 16), and one had a larger BP3/BP5 duplication (Patient 4). Neither individual with larger duplications had any additional phenotypes that may be due to the inclusion of additional genes on chromosome 15q. This is consistent in the literature for both 15q13.3 duplications and deletions, which has indicated that, although there is variable expressivity for all the CNVs, the size of the copy number change does not seem to add or alter phenotypes (Gillentine \& Schaaf, 2015). This has been suggested as further evidence for $C H R N A 7$ as a strong candidate gene, as it is the only gene fully included in the small D-CHRNA7-LCR/BP5 CNVs.

Another possible explanation for the variable expressivity observed are single nucleotide variants and/or CNVs in modifier genes contributing to the overall phenotype, in conjunction with copy number of $C H R N A 7$. A strong candidate for such a modifier is CHRFAM7A, the human specific fusion gene of $C H R N A 7$ and $F A M 7 A$, which has been suggested to act as a dominant negative regulator of $C H R N A 7$ (Sinkus et al., 2015). CHRFAM7A is copy variable and polymorphic, with a 2 base pair deletion polymorphism being negatively associated with idiopathic generalized epilepsy and positively associated with schizophrenia (Flomen et al., 2006; Rozycka et al., 2013). Therefore, it is possible that interactions among $C H R N A 7$ and $C H R F A M 7 A$, or other potential modifying genes, occur in human neurons and may affect the phenotypes observed in patients with CHRNA7CNVs. However, this has yet to be explored in human cells.

Five patients have additional CNVs, which may contribute to their phenotype, as suggested by the two-hit hypothesis for ID/DD (Girirajan et al., 2012) (Supplementary Table S1). We chose to include these individuals with additional CNVs, including one with a 22q11.21 deletion, as secondary CNVs were not considered an exclusion criterion for this study. However, we did not observe a significant difference between probands with additional CNVs and probands with only 15q13.3 duplications, although our sample size of proband with additional CNVs was small (Fig S5). Of note, Patient 7 carrying a 22q11.21 deletion, which is diagnostic of Velocardiofacial Syndrome (VCFS), did have features of VCFS, including heart problems and dysmorphic features. 
Our cohort of patients has a high prevalence of ASD, with 35\% $(n=6)$ having a strict diagnosis of ASD, meeting cutoffs on both the ADOS-2 and the ADI-R (Supplementary Table S5, Fig. 6). Furthermore, an additional 30\% $(n=5)$ met the criteria for the ADOS-2 or ADI-R, but not both, indicating that autistic behaviors are prevalent in this group of patients. Based on this study, the prevalence of ASD among CHRNA7 duplication probands is higher than in most other genetic syndromes, such as 1q21.1 distal deletions (10\%), 16p11.2 proximal deletions (27\%), and even CHRNA7 deletions (31\%), but is lower than some genetic syndromes, such as 1q21.1 duplications (41\%) (Bernier et al., 2015; Hanson et al., 2010; Ziats et al., 2016). This emphasizes the need for parents of children with CHRNA7 duplications to be aware of potential autistic behaviors. Furthermore, the variable expressivity within individuals with ASD who carry a CHRNA7 duplication makes these ASD diagnoses more complex, as it appears that $C H R N A 7$ copy number changes do not result in a distinct subset of autistic features. Additionally, it is probable that $C H R N A 7$ duplications represent a common CNV among individuals with ASD, however this would have to be explored in a cohort of only children with ASD, using a CMA sensitive enough to detect these duplications reliably.

Other neuropsychiatric features are present in a subset of our patients, including ADHD (44\%), in which CHRNA 7 duplications have been previously identified in $1.25 \%$ of patients (Williams et al., 2010), and mood disorders (17\%). ADHD had considerable comorbidity with ASD, with half of those diagnosed with ASD also reporting ADHD and/or hyperactivity. Two probands who did not meet both ADI-R and ADOS criteria for autism also reported ADHD. Interestingly, schizophrenia was not reported in any of our patients, but was reported in one parental carrier. Schizophrenia has been identified in about $25 \%$ of previously reported individuals with $15 \mathrm{q} 13.3$ duplications, majority of which were DCHRNA7-LCR/BP5 gains (Gillentine \& Schaaf, 2015). The lack of schizophrenia among our cohort may be due to the age of our probands (all 14 years of age or younger), or different ascertainment bias in this cohort when compared to previous studies.

In comparison to individuals with $15 \mathrm{q} 13.3$ microdeletions, the phenotypes associated with $15 q 13.3$ duplications are milder, with features common in 15q13.3 microdeletion patients, including seizures/epilepsy, being considerably less prevalent in 15q13.3 microduplication probands. Our cohort of $15 \mathrm{q} 13.3$ duplication probands have statistically significantly higher FSRIQ scores $(\mathrm{p}=0.0175)$ and NVRIQ scores $(\mathrm{p}=0.0088)$ than a recently phenotyped cohort of CHRNA7 deletion individuals (Fig. 8; Ziats et al., 2016). The difference in severity of similar phenotypes is highlighted by the ABAS-II and BASC-2 scores, which deviated from the average norms in both cohorts. All measures that were significantly different between the current cohort and the 15q13.3 deletion cohort were closer to the normal average for duplication probands. This suggests that gains in copy number of $C H R N A 7$ may have less severe pathogenic consequences than losses in most individuals. This trend is consistent with the literature (Gillentine \& Schaaf, 2015; Szafranski et al., 2010). This is further suggested by the prevalence of small CHRNA7 duplication versus reciprocal deletions. D-CHRNA7LCR/BP5 mediated duplications occur approximately ten fold more often in CMA samples than D-CHRNA7-LCR/BP5 mediated deletions. This may suggest that there is selection against these smaller deletions, supporting the notion that duplications are more tolerable due to their less severe phenotypes. However, the higher prevalence of duplications than 
deletions may also be due to the complex architecture of the region, with various rearrangements, such as inversions promoted by NAHR, predisposing to particular CNVs. Interestingly, triplications of $C H R N A 7$ result in phenotypes remarkably similar to those seen in duplication patients, including ID/DD and ADHD, although very few cases have been reported (Gillentine \& Schaaf, 2015; Soler-Alfonso et al., 2014). While it appears that CHRNA7 duplication probands have a less severe phenotype, it also is apparent that their phenotypes may be more developmental disorders focused, including ASD, ADHD, and mood disorders. This highlights that the mechanisms and functional consequences of these gains and reciprocal deletions may be differing in certain ways, most likely requiring different therapeutic approaches.

All reported small $C H R N A 7$ duplications have been inherited. In this study, again, all of the CHRNA7 gains for which we could identify inheritance were inherited. While not formally evaluated in this study, over half of those carrier parents had reported neuropsychiatric phenotypes themselves, including ID, learning disabilities, depression, bipolar disorder, and schizophrenia (Figs. 2, S1, Table 2). However, the number of CHRNA7 duplications inherited from parents carrying the CNV but not reporting any phenotypes $(\mathrm{n}=6,35 \%)$ highlights the incomplete penetrance that $C H R N A 7$ gains exhibit. Furthermore, several parents without $C H R N A 7$ duplications reported neuropsychiatric phenotypes including depression, ADHD, and anxiety. With most parent sets either having both parents report neuropsychiatric phenotypes ( $\mathrm{n}=8$ parental sets, $47 \%$ ) or neither parent reporting neuropsychiatric phenotypes ( $\mathrm{n}=6,35 \%)$, it is possible that assortative mating may be occurring among this population, which has been observed among individuals with neuropsychiatric disorders previously (Mathews \& Reus, 2001). This may support the notion of modifiers impacting the variable expressivity of these CNVs, as probands may have inherited a CHRNA7 duplication from one parent and an additional neuropsychiatric involved modifier from the other parent. Of note, all but one family reported parents being fully capable of taking care of their children, with only one maternal carrier with intellectual disability living with and receiving care from the proband's paternal grandmother. Since half of these parents were carriers themselves, phenotypes associated with CHRNA7 duplications may not be severe enough to significantly reduce independence in some individuals, although a study focusing on more adults with these gains would be necessary to make a significant conclusion. This is in contrast to the potential long-term phenotypes of CHRNA7 deletions, also typically inherited, which may be more detrimental for independent living, as a third of the children in a similar study of CHRNA7 deletion probands were adopted after being placed in foster care, suggesting the possibility that their biological parents had neuropsychiatric phenotypes preventing them from successfully taking care of their children (Ziats et al., 2016). Additionally, the evidence of adopted children with CHRNA7 deletions exhibiting a similar phenotypic spectrum to those not adopted suggests that $C H R N A 7$ is playing a role in their phenotype, as opposed to parental neuropsychiatric disorders having an influence. We did not observe any significant differences between probands with parents who reported neuropsychiatric phenotypes and those who did not (data not shown). As $C H R N A 7$ is dosage sensitive, this supports the conclusion that $C H R N A 7$ duplications are playing a role in the phenotypes of this cohort of patients and that they are not heavily influenced by parental diagnoses. 
Identified in $0.81 \%$ of samples submitted for clinical microarray at the BMGL and at Signature Genomic Laboratories, 15q13.3 microduplications represent the most common genetic alterations identified by CMA in individuals with neuropsychiatric disease. All of our cohort were ascertained from samples tested by CMA, which are very likely to all have neurocognitive or neuropsychiatric features, as they are clinical samples. However, the same microduplications are seen at high prevalence in control populations as well. It is important to consider that the definition of "control" populations may vary among studies. It has been shown that CNVs may have negative effects in the general population, including cognitive impairments and other neuropsychiatric features, however they may not result in clinical attention (Männik et al., 2015; Stefansson et al., 2014). This may contribute to "control" populations having potential for unreported phenotypes, such as ADHD, mood disorders, borderline IQ, and other cognitive or behavioral phenotypes that may not be addressed clinically. This may be especially true for CNVs that can result in mild, variable impairments, such as 15q13.3 duplications. Strict inclusion and exclusion criteria would have to be defined for "control individuals" used to accurately assess the prevalence of CHRNA7 duplications in the general population.

Our study has several limitations, primarily surrounding ascertainment bias. While we have identified a pattern of behavioral and cognitive phenotypes among probands with $C H R N A 7$ duplications, we are limited by enrolling only individuals who had previously been tested by CMA analysis for clinical indications. Clinical CMA is considered a first tier test in the evaluation of individuals with DD, ID, congenital anomalies, and ASD. Thus, probands with these phenotypes are inherently overrepresented in any study that enrolls based on previously identified CNVs. With this inherent overrepresentation and the similar frequency of these duplications in control populations, the phenotypes reported for probands in this study are not fully representative of all individuals with $15 q 13.3$ duplications in the population. Attempts should be made to overcome this ascertainment bias, for example by phenotyping individuals who had been identified to carry $C H R N A 7$ duplications based on prenatal CMA or by phenotyping siblings and/or "control" individuals identified to carry a CHRNA7 gain. In addition, our study is limited by the age range of individuals enrolled. Neuropsychiatric phenotypes that have age-dependent penetrance, such as schizophrenia or bipolar disorder, are most likely underrepresented in this cohort.

The genotype-to-phenotype approach and comprehensive assessment of cognition and behavior in our study may provide information that is useful for providing counseling and anticipatory guidance to individuals who were diagnosed with these overlapping CNVs based on postnatal CMA testing. By establishing the pathogenicity of duplications of CHRNA7, it is now possible to provide more thorough and confident guidance for patients and families, allowing them to better address their or their child's specific needs. However, the incomplete penetrance and variable expressivity associated with these CNVs leave a great level of uncertainty. While genetic testing for incompletely penetrant and variably expressed CNVs can answer part of a patient's phenotype, it is important to consider that variable phenotypes may require different therapeutic approaches. Future studies need to address the biological consequences and potential modifiers of these gains in CHRNA7, all of which will be important in developing therapeutics and in understanding how this genomic region contributes to human cognition and human behavior. 


\section{Supplementary Material}

Refer to Web version on PubMed Central for supplementary material.

\section{Acknowledgments}

This work was generously supported by the Doris Duke Charitable Foundation Grant \#2011034. The project was supported in part by IDDRC grant number 1U54 HD083092 from the Eunice Kennedy Shriver National Institute of Child Health \& Human Development. Cores: Tissue culture core, translational core. Miss Gillentine was supported by Grant Number T32GM008307 from the National Institute of General Medical Sciences. The content is solely the responsibility of the authors and does not necessarily represent the official views of the National Institute of General Medical Sciences or the National Institutes of Health. Dr. Schaaf was generously supported by the Joan and Stanford Alexander Family.

Funding: This study was funded by the Doris Duke Charitable Foundation Grant \#2011034. The project was supported in part by IDDRC grant number 1U54 HD083092 from the Eunice Kennedy Shriver National Institute of Child Health \& Human Development. Miss Gillentine was supported by Grant Number T32GM008307 from the National Institute of General Medical Sciences. Dr. Schaaf was generously supported by the Joan and Stanford Alexander Family.

\section{References}

Bernier R, Steinman KJ, Reilly B, Wallace AS, Sherr EH, Pojman N, ... Chung WK. Clinical phenotype of the recurrent 1q21.1 copy-number variant. Genetics in medicine : official journal of the American College of Medical Genetics. 2015; doi: 10.1038/gim.2015.78

Flomen R, Collier D, Osborne S, Munro J, Breen G, Clair \{St. Association study of CHRFAM7A copy number and $2 \mathrm{bp}$ deletion polymorphisms with schizophrenia and bipolar affective disorder. American journal of medical genetics. Part B, Neuropsychiatric genetics : the official publication of the International Society of Psychiatric Genetics. 2006; 141B(6):571-5. DOI: 10.1002/ajmg.b. 30306

Gillentine MA, Schaaf CP. The human clinical phenotypes of altered CHRNA7 copy number. Biochemical pharmacology. 2015; 97(4):352-62. DOI: 10.1016/j.bcp.2015.06.012 [PubMed: 26095975]

Girirajan S, Eichler E. Phenotypic variability and genetic susceptibility to genomic disorders. Human molecular genetics. 2010; 19(R2):R176-87. DOI: 10.1093/hmg/ddq366 [PubMed: 20807775]

Girirajan S, Rosenfeld JA, Coe BP, Parikh S, Friedman N, Goldstein A, ... Eichler EE. Phenotypic heterogeneity of genomic disorders and rare copy-number variants. The New England journal of medicine. 2012; 367(14):1321-31. DOI: 10.1056/NEJMoa1200395 [PubMed: 22970919]

Hanson E, Nasir RH, Fong A, Lian A, Hundley R, Shen Y, ... Miller DT. Cognitive and behavioral characterization of $16 \mathrm{p} 11.2$ deletion syndrome. Journal of developmental and behavioral pediatrics : JDBP. 2010; 31(8):649-57. DOI: 10.1097/DBP.0b013e3181ea50ed [PubMed: 20613623]

Helbig I, Mefford HC, Sharp AJ, Guipponi M, Fichera M, Franke A, ... Sander T. 15q13.3 microdeletions increase risk of idiopathic generalized epilepsy. Nature genetics. 2009; 41(2):160-2. DOI: 10.1038/ng.292 [PubMed: 19136953]

Lowther C, Costain G, Stavropoulos D, Melvin R, Silversides C, Andrade D, ... Bassett A. Delineating the 15 q13.3 microdeletion phenotype: a case series and comprehensive review of the literature. Genetics in medicine : official journal of the American College of Medical Genetics. n.d Feb.:1-9. DOI: $10.1038 /$ gim.2014.83

Männik K, Mägi R, Macé A, Cole B, Guyatt AL, Shihab HA, ... Reymond A. Copy number variations and cognitive phenotypes in unselected populations. JAMA. 2015; 313(20):2044-54. DOI: 10.1001/ jama.2015.4845 [PubMed: 26010633]

Mathews CA, Reus VI. Assortative mating in the affective disorders: a systematic review and metaanalysis. Comprehensive psychiatry. 2001; 42(4):257-62. DOI: 10.1053/comp.2001.24575 [PubMed: 11458299]

Rozycka A, Dorszewska J, Steinborn B, Lianeri M, Winczewska-Wiktor A, Sniezawska A, ... Jagodzinski PP. Association study of the 2-bp deletion polymorphism in exon 6 of the 
CHRFAM7A gene with idiopathic generalized epilepsy. DNA and cell biology. 2013; 32(11):6407. DOI: 10.1089/dna.2012.1880 [PubMed: 24024466]

Shinawi M, Schaaf CP, Bhatt SS, Xia Z, Patel A, Cheung SW, ... Stankiewicz P. A small recurrent deletion within $15 \mathrm{q} 13.3$ is associated with a range of neurodevelopmental phenotypes. Nature genetics. 2009; 41(12):1269-71. DOI: 10.1038/ng.481 [PubMed: 19898479]

Sinkus ML, Graw S, Freedman R, Ross RG, Lester HA, Leonard S. The human CHRNA7 and CHRFAM7A genes: A review of the genetics, regulation, and function. Neuropharmacology. 2015; 96(Pt B):274-88. DOI: 10.1016/j.neuropharm.2015.02.006 [PubMed: 25701707]

Soler-Alfonso C, Carvalho C, Ge J, Roney E, Bader P, Kolodziejska K, ... Schaaf C. CHRNA7 triplication associated with cognitive impairment and neuropsychiatric phenotypes in a threegeneration pedigree. European journal of human genetics : EJHG. n.d; (October 2013):1-6. DOI: 10.1038/ejhg.2013.302

Stefansson H, Meyer-Lindenberg A, Steinberg S, Magnusdottir B, Morgen K, Arnarsdottir S, ... Stefansson K. CNVs conferring risk of autism or schizophrenia affect cognition in controls. Nature. 2014; 505(7483):361-6. DOI: 10.1038/nature12818 [PubMed: 24352232]

Szafranski P, Schaaf CP, Person RE, Gibson IB, Xia Z, Mahadevan S, ... Stankiewicz P. Structures and molecular mechanisms for common 15q13.3 microduplications involving CHRNA7: benign or pathological? Human mutation. 2010; 31(7):840-50. DOI: 10.1002/humu.21284 [PubMed: 20506139]

Williams NM, Franke B, Mick E, Anney RJ, Freitag CM, Gill M, ... Faraone SV. Genome-wide analysis of copy number variants in attention deficit hyperactivity disorder: the role of rare variants and duplications at 15q13.3. The American journal of psychiatry. 2012; 169(2):195-204. DOI: 10.1176/appi.ajp.2011.11060822 [PubMed: 22420048]

Williams NM, Zaharieva I, Martin A, Langley K, Mantripragada K, Fossdal R, ... Thapar A. Rare chromosomal deletions and duplications in attention-deficit hyperactivity disorder: a genome-wide analysis. Lancet. 2010; 376(9750):1401-8. DOI: 10.1016/S0140-6736(10)61109-9 [PubMed: 20888040]

Ziats MN, Goin-Kochel RP, Berry LN, Ali M, Ge J, Guffey D, ... Schaaf CP. The complex behavioral phenotype of 15q13.3 microdeletion syndrome. Genetics in medicine : official journal of the American College of Medical Genetics. 2016; doi: 10.1038/gim.2016.9 


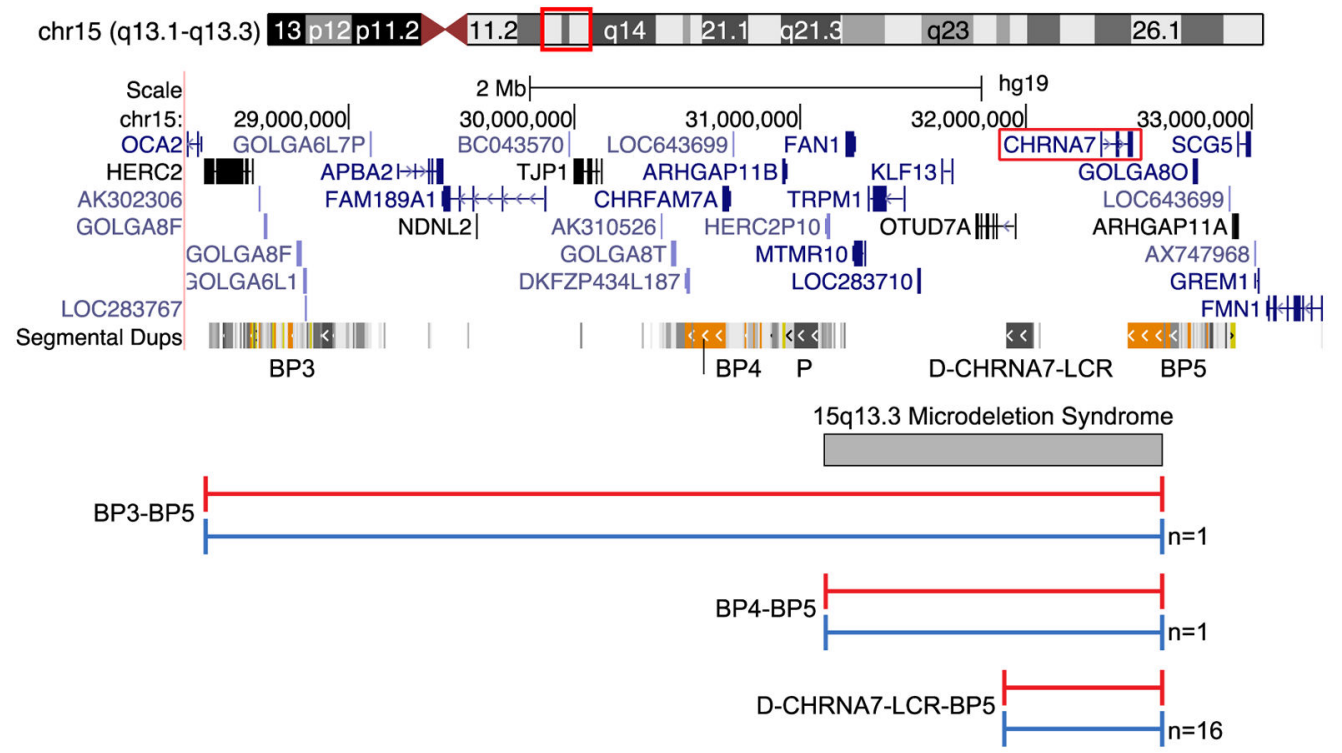

Figure 1.

Genomic architecture and copy number variants at chromosome 15q13.3. Segmental duplications (dups) show low copy repeat (LCR) elements clustering into breakpoints 3-5 (BP3-BP5) and the proximal (P) and distal (D-CHRNA7-LCR) CHRNA7LCR elements. $15 q 13.3$ microdeletion syndrome DECIPHER coordinates are shown in grey. CHRNA7 is highlighted in a red box, as is the locus on chromosome 15. Deletions (red) and duplications (blue) are shown with the number of probands in this study indicated next to the appropriate duplication. All probands had a duplication spanning D-CHRNA7-LCR/BP5 except Patient 4 (BP3/BP5) and Patient 16 (BP4/BP5). 

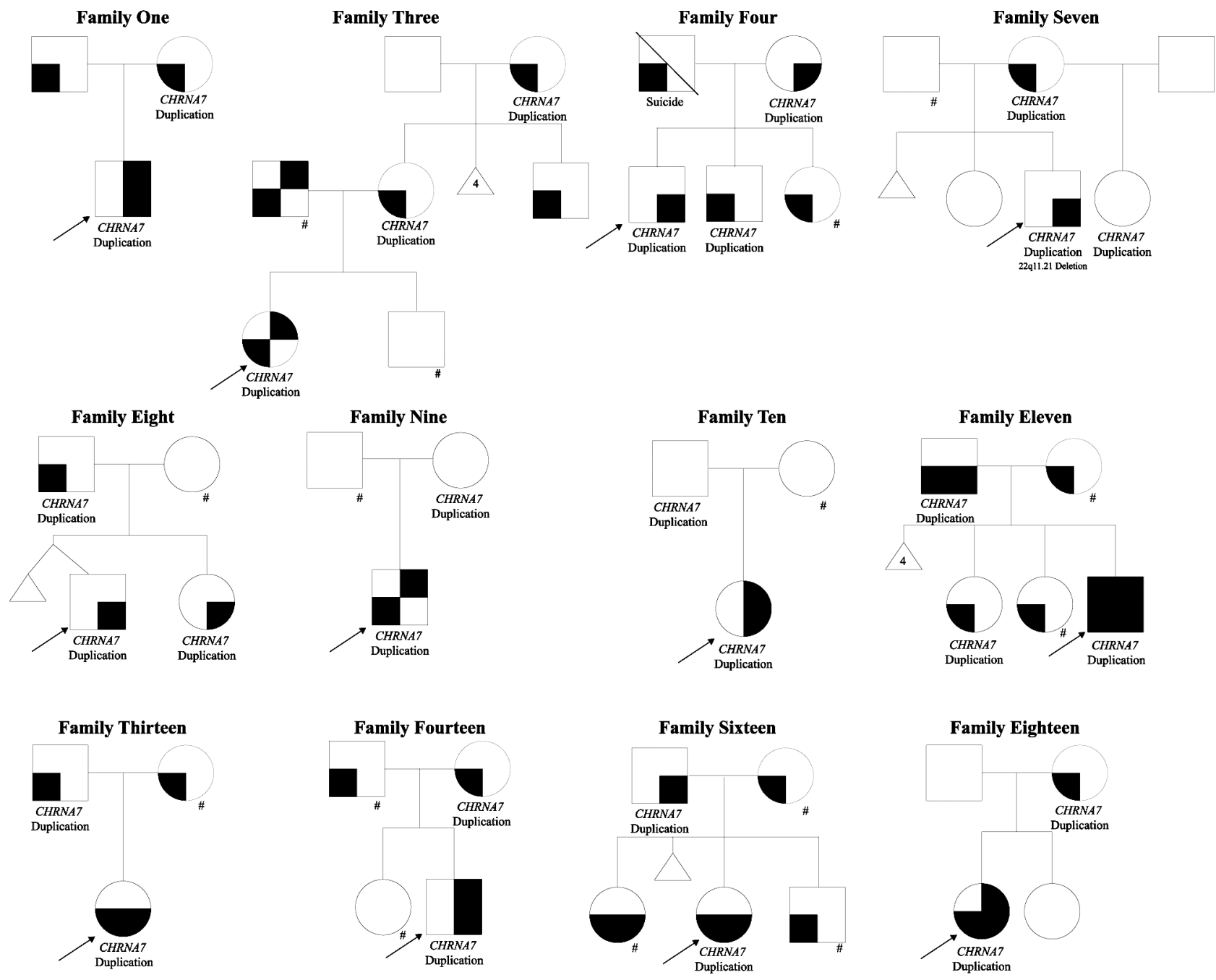

Figure 2.

Pedigrees of all CHRNA7 duplication probands who provided consent to have this information published. Incomplete penetrance was observed with these duplications as well as variable expressivity. Arrow indicates proband. The presence of a CHRNA7 duplication is noted under each individual who positively tested for the CNV. \# indicates individuals who tested negative for the duplication. Shading indicates the following: top left: seizures, top right: ASD, bottom left: other neuropsychiatric conditions, bottom right: cognitive deficits. 


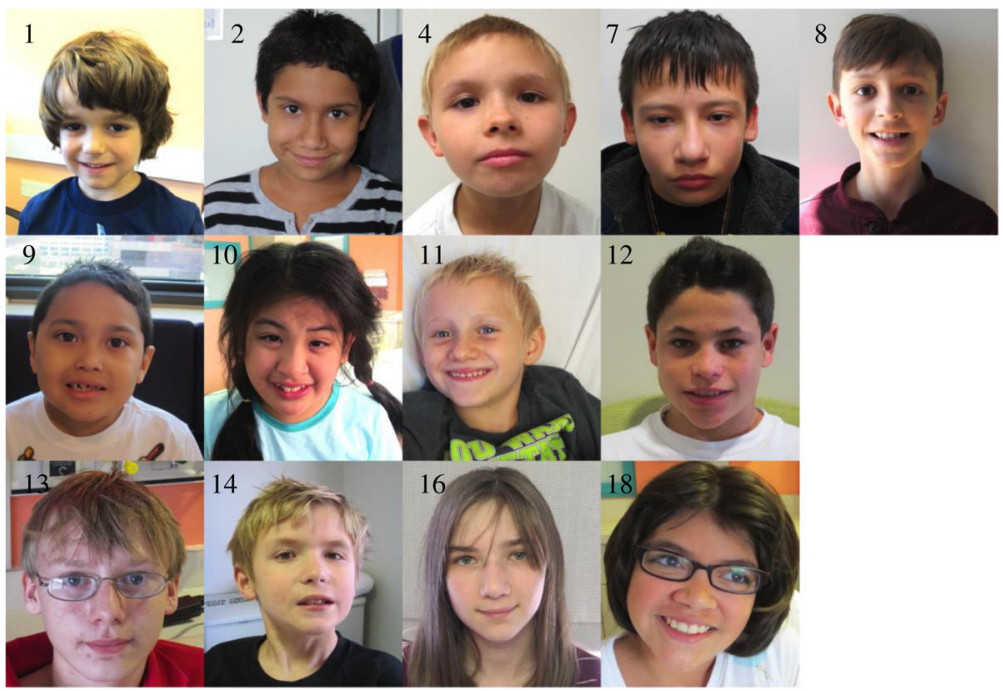

Figure 3.

Facial features of 13 of 18 reported probands. No consistent dysmorphic features were appreciated. 

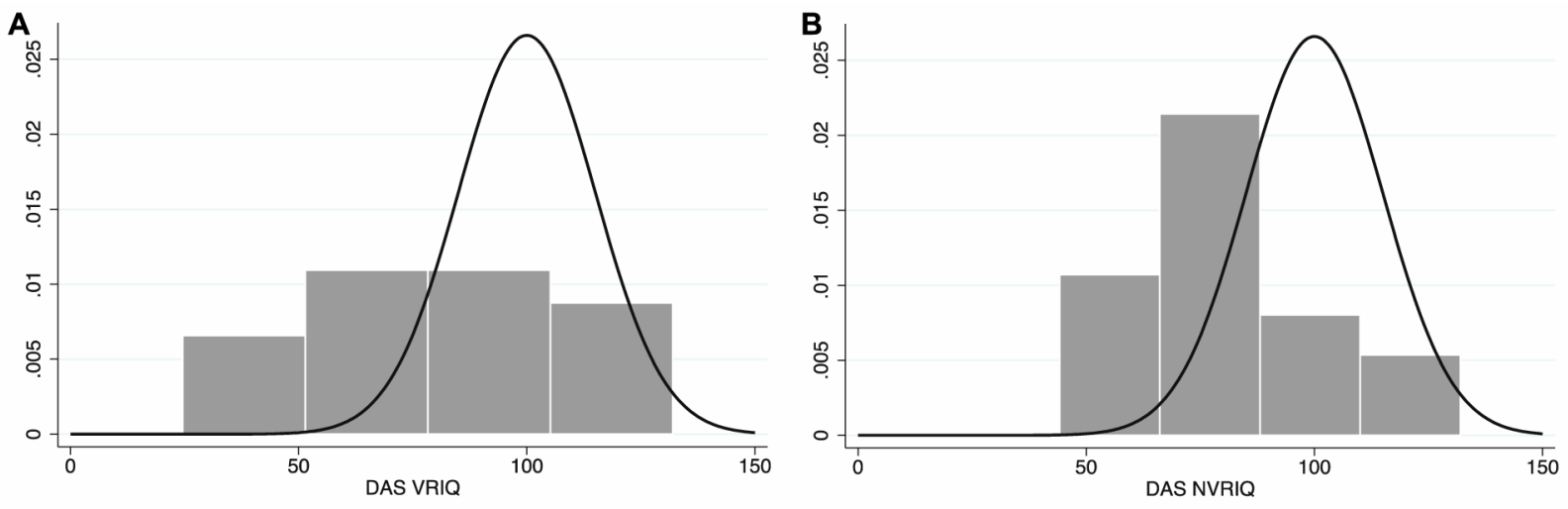

C

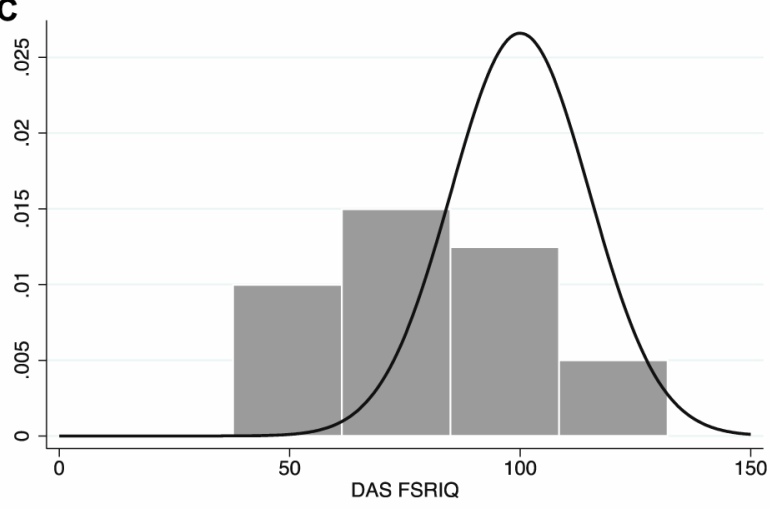

D

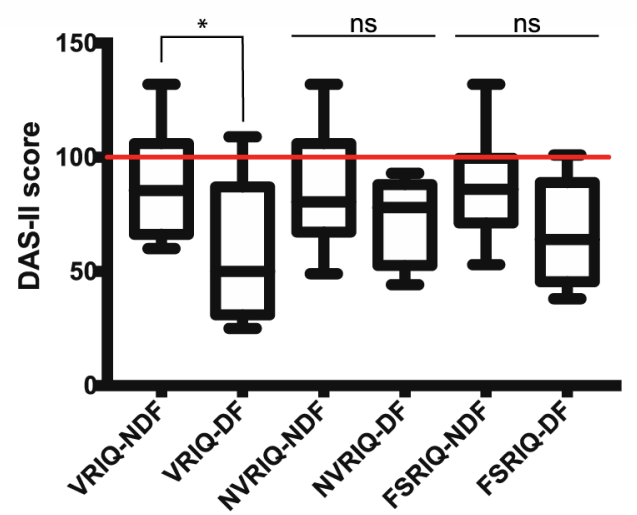

Figure 4.

Histograms of the Differential Abilities Scale II (DAS-II) composite scores of CHRNA7 duplication probands. Curve showing the normative scores using a normal distribution with a mean of 100 and standard deviation of 15 is shown. All measurements (A) Verbal ratio IQ (VRIQ) composite scores, (B) Nonverbal ratio IQ (NVRIQ) composite scores, and (C) Full scale ratio IQ (FSRIQ) composite scores were decreased compared to the norm. 

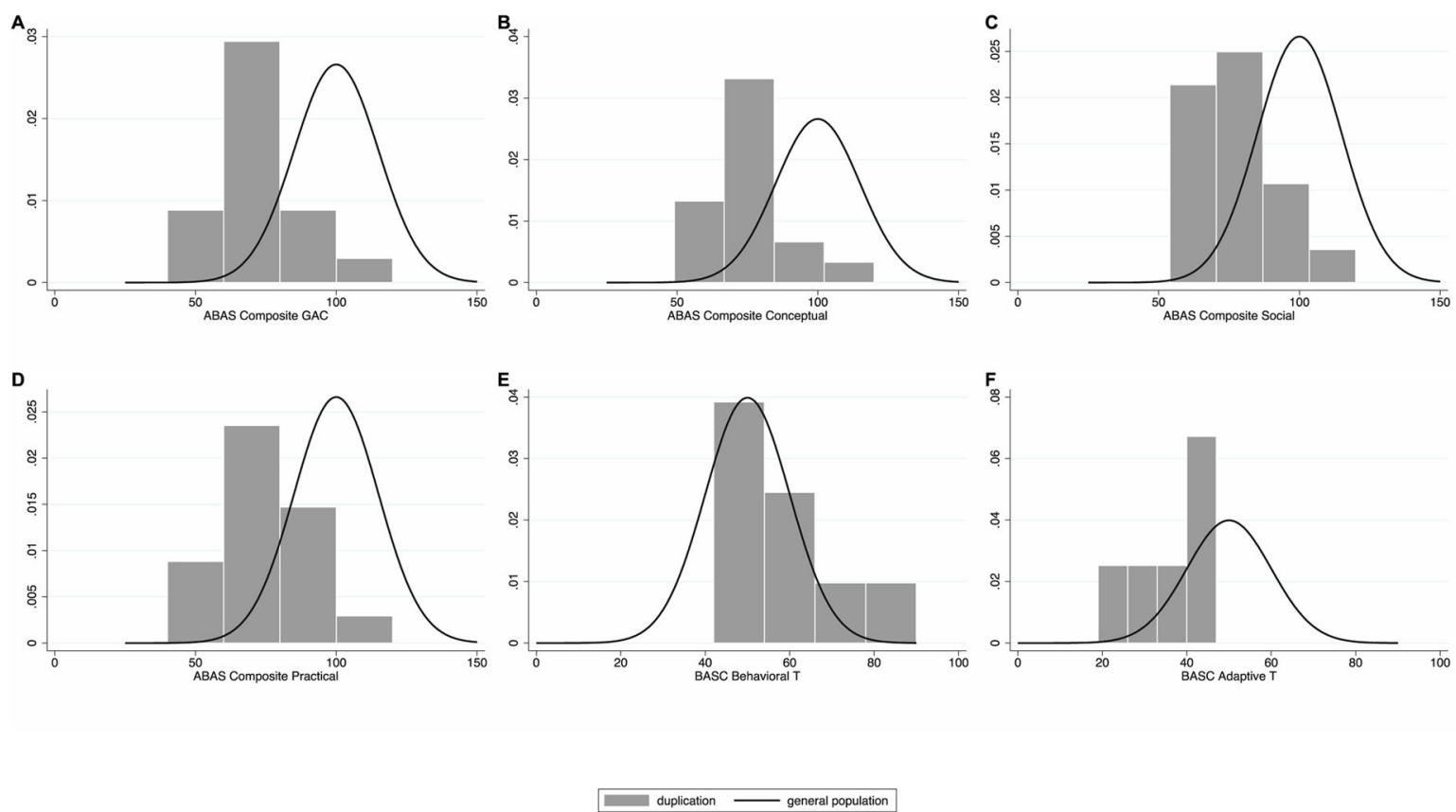

Figure 5.

Histograms of the clinically significant measures in the Adaptive Behavior Assessment System, Version II (ABAS-II) of CHRNA7 duplication probands. Curve showing the normative scores using a normal distribution with a mean of 100 and standard deviation of 15 is shown. All measures (A) Average general adaptive composite score (GAC), (B) Average conceptual composite score, (C) Average social composite score, and (D) Average practical composite score clinically different compared to the norm. 


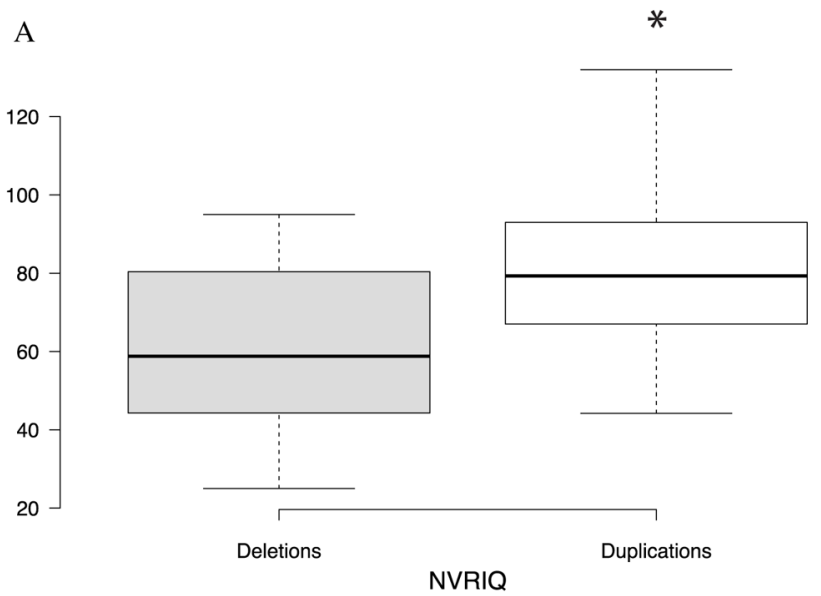

B

Figure 6.

Histograms of the clinically significant measures in the Behavior Assessment System for Children, Edition 2 (BASC-2) results and subscale measures. (A) Average behavior T score. BASC-2 curve showing the normative scores using a normal distribution with a mean of 50 and standard deviation of 10 is shown. (B) Average adaptive T score. BASC-2 curve showing the normative scores using a normal distribution with a mean of 50 and standard deviation of 10 is shown. (C) BASC-2 Average Subscale T scores with standard deviation. Average normative score of 50 is indicated by red line. 


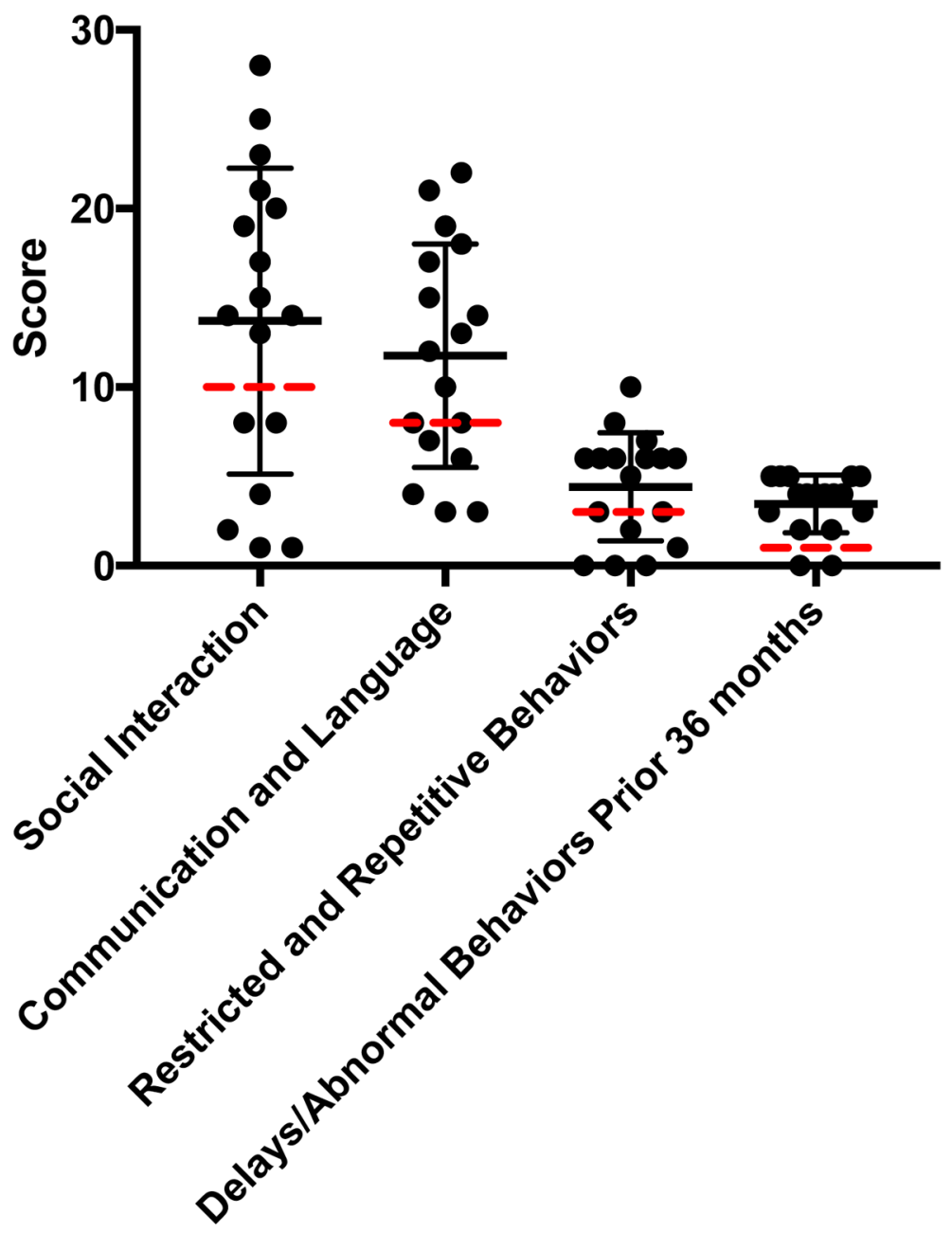

ADI-R Domain

Figure 7. Autism Diagnostic Interview-Revised

(ADI-R) measures of $C H R N A 7$ duplication probands. Red dashed line indicates cut off score to meet ADI-R criteria (Cutoff Scores: 10 for Social Interactions, 8 for

Communication and Language, 3 for Restricted and Repetitive Behaviors, and 1 for Delays/ Abnormal Behaviors Prior to 36 months). ADI-R scores were variable, with a subset of probands meeting cut off scores. 

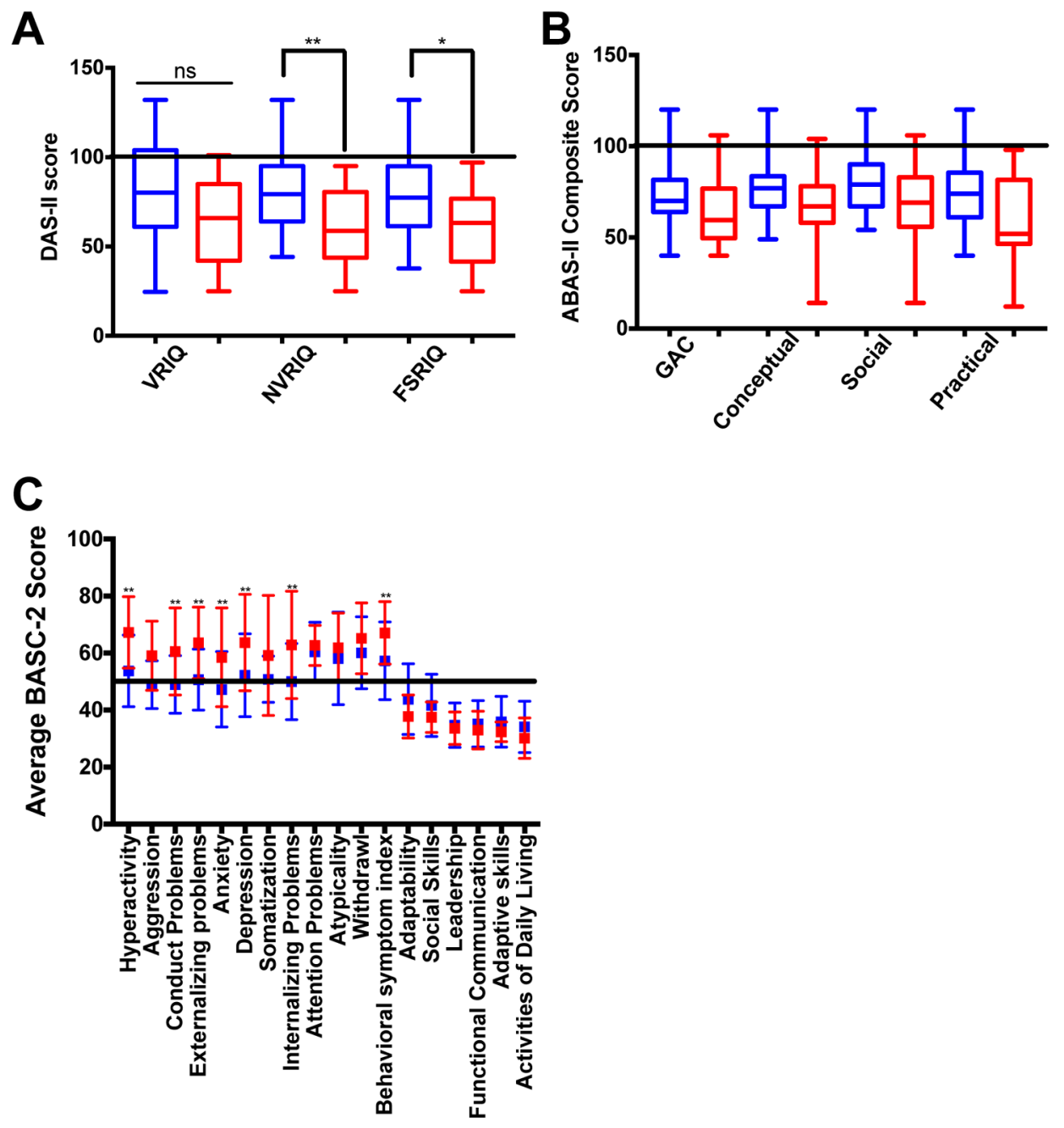

Figure 8.

Comparative scores of $C H R N A 7$ duplication and deletion probands. (A) VRIQ, NVRIQ and FSRIQ measurements of 15q13.3 duplication $(n=17)$ and deletion $(n=18)$ probands.

Duplication probands had significantly higher NVRIQ measurements $(\mathrm{p}=0.0088)$ and FSRIQ measurements ( $\mathrm{p}=0.0175$ ) than deletion probands. (B) Adaptive Behavior Assessment System, Version II (ABAS-II) scores. No significant differences were observed. (C) Behavior Assessment System for Children, Edition 2 (BASC-2) subscale measures. Significant differences were observed for Hyperactivity (0.0043), Conduct Problems (0.0169), Externalizing Problems $(\mathrm{p}=0.0034)$, Anxiety $(\mathrm{p}=0.0433)$, Depression $(0.0449)$, Internalizing Problems ( $\mathrm{p}=0.0299)$, and the Behavioral Symptom Index (0.0311). Center lines show the medians; box limits indicate the 25 th and 75 th percentiles as determined by Prism; whiskers extend 1.5 times the interquartile range from the 25 th and 75 th percentiles. 


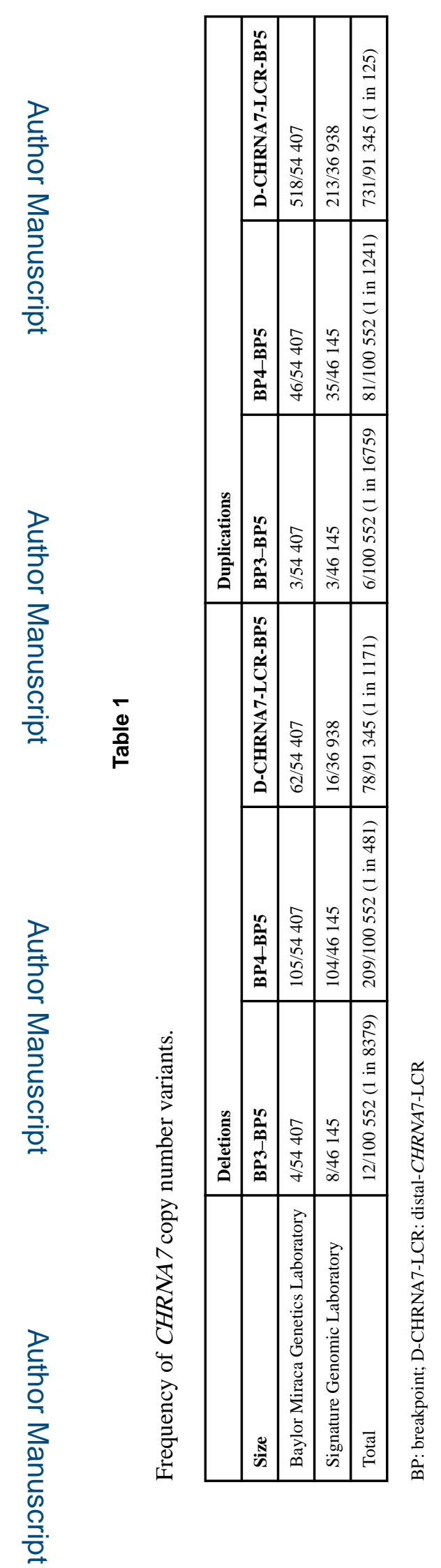

J Autism Dev Disord. Author manuscript; available in PMC 2018 March 01. 


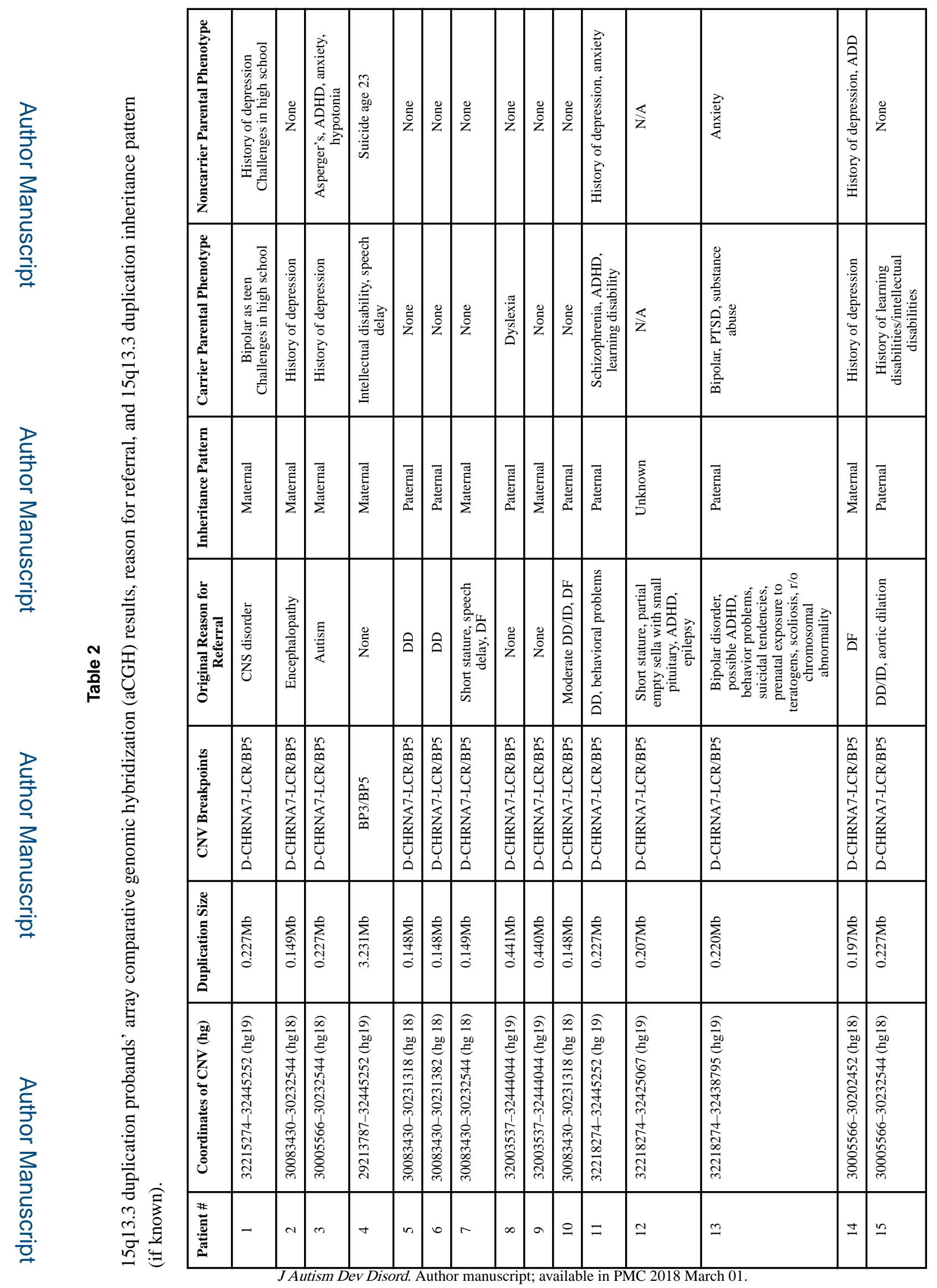




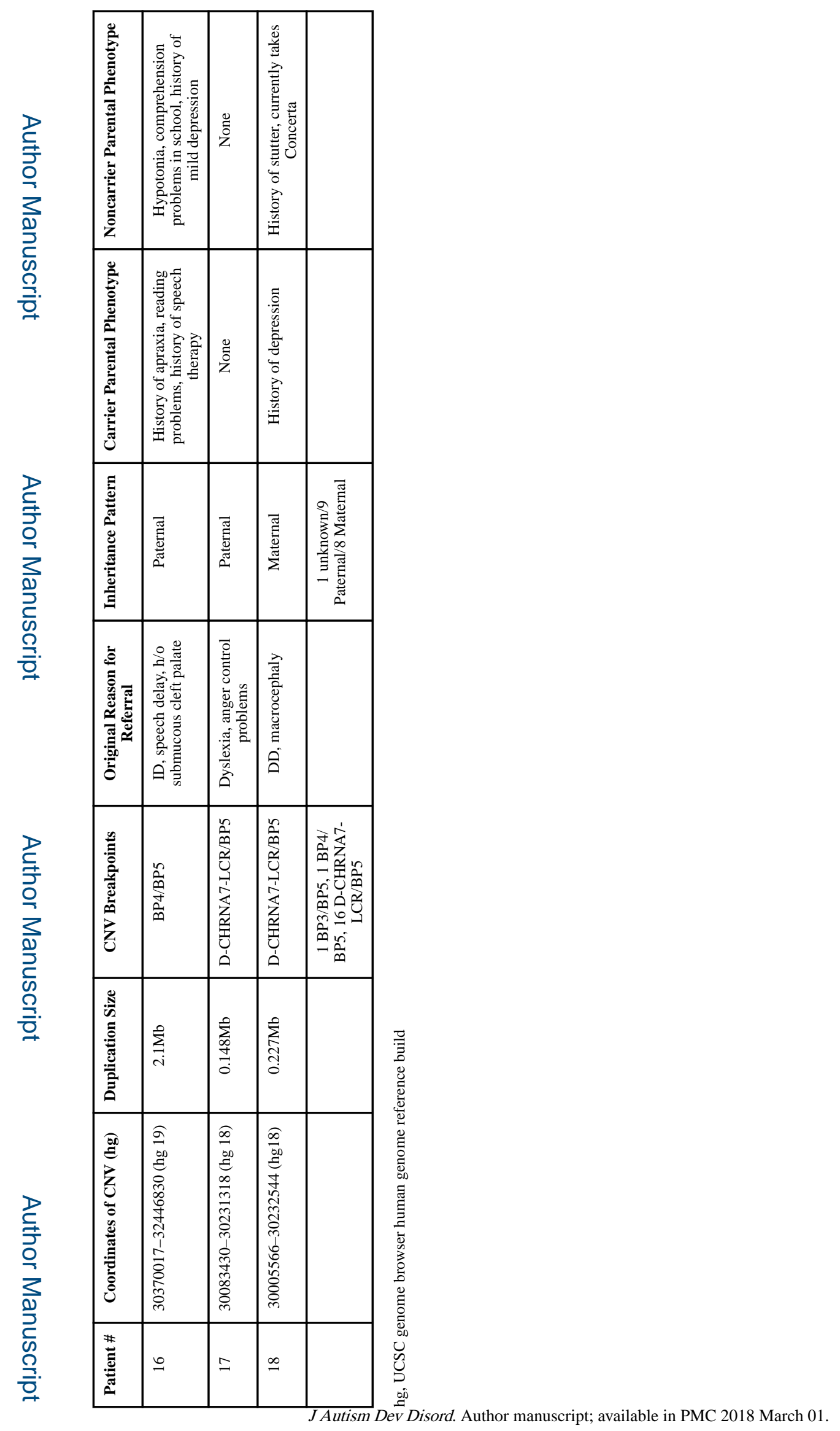




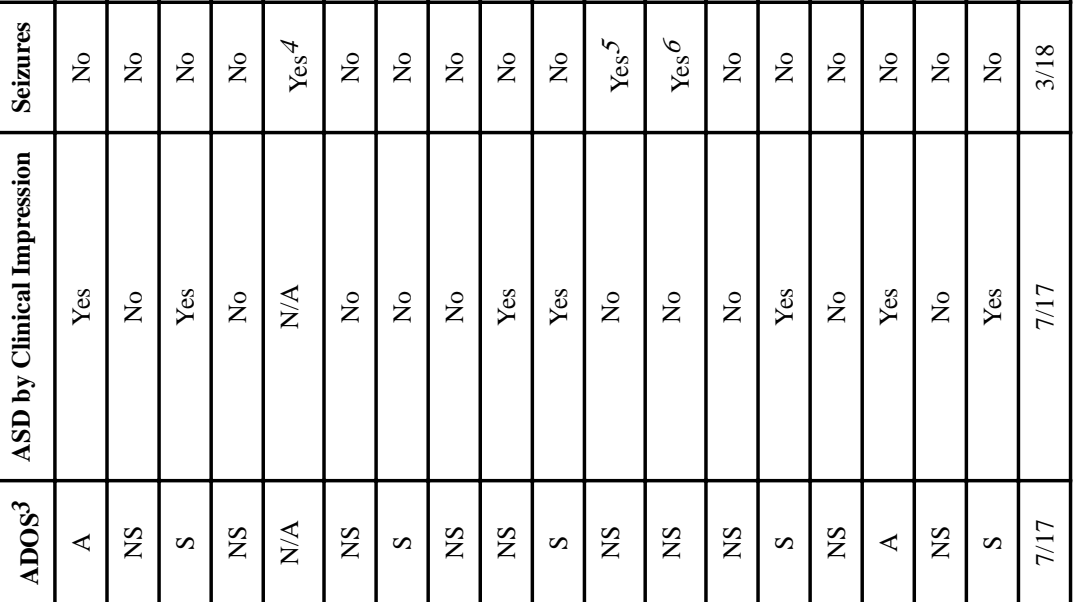

$\frac{m}{\infty}$

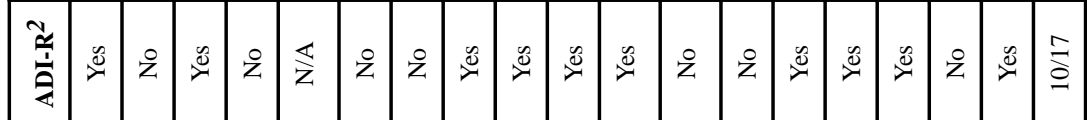

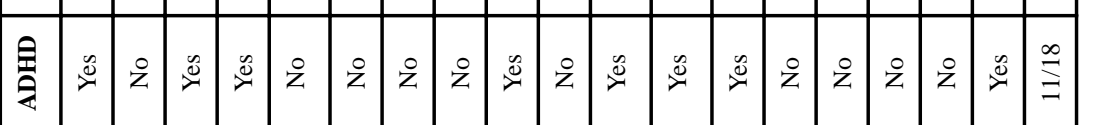

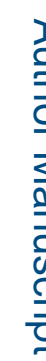

롤
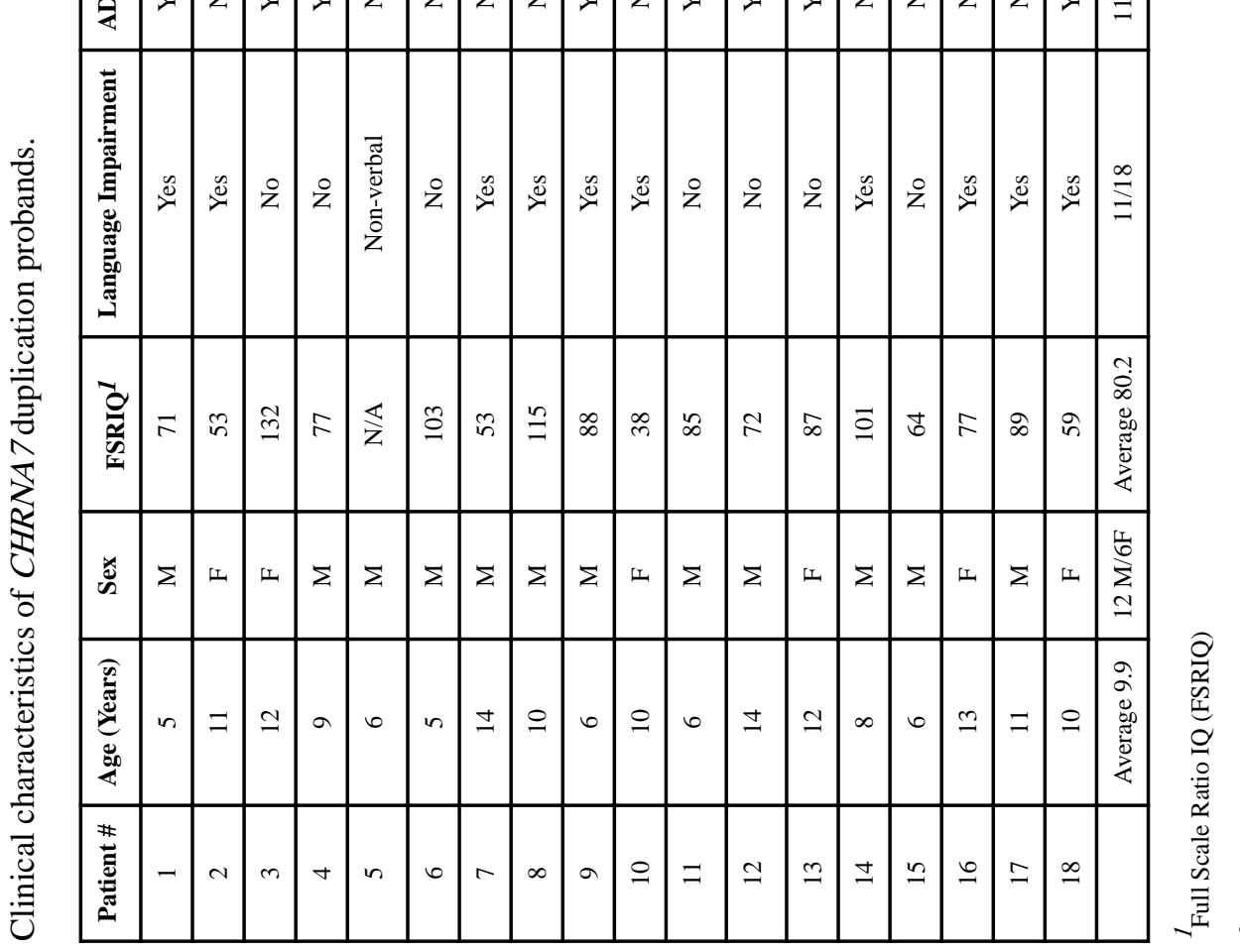
